\title{
A Bound on the Ratio between the Packing and Covering Densities of a Convex Body
}

\author{
E. H. Smith \\ MCIS Department, Jacksonville State University, \\ Jacksonville, AL 36365, USA \\ esmith@jsucc.jsu.edu
}

\begin{abstract}
It is shown that if $K$ is a compact convex set which is centrally symmetric and has a nonempty interior, then the density of the tightest lattice packing with copies of $K$ in Euclidean 3-space divided by the density of the thinnest lattice covering of Euclidean 3 -space with copies of $K$ is greater than or equal to $\frac{1}{4}$. It is likely this bound can be improved, though not beyond approximately $\frac{1}{2}$.
\end{abstract}

\section{Introduction}

A convex body is a compact convex set having a nonempty interior. In the context of this paper, we are only concerned with convex bodies in Euclidean 3-space $\left(E^{3}\right)$ and in the Euclidean plane $\left(E^{2}\right)$ and we denote by $K$ an arbitrary convex body. A convex body in the plane is referred to as a convex disk. $K$ is centrally symmetric if there exists a point $o$ in $K$ such that $o$ is the midpoint of every chord of $K$ which contains $o$.

A packing with copies of $K$ is a family $\left\{K_{i}\right\}$ of sets congruent to $K$ whose interiors are mutually disjoint. A packing with copies of $K$ is saturated if it cannot be augmented with an additional copy of $K$ while remaining a packing. A covering with copies of $K$ is a family $\left\{K_{i}\right\}$ of sets congruent to $K$ whose union is the entire space. A family $\left\{K_{i}\right\}$ which is both a packing and a covering is called a tiling. Any convex body which admits a tiling is called a convex tile.

Suppose $T$ is a tile and $T$ contains (respectively is contained in) $K$. If $\left\{T_{i}\right\}$ is a tiling with copies of $T$, then each $T_{i}$ contains (is contained in) a copy $K_{i}$ of $K$. Then $\left\{K_{i}\right\}$ is a packing (covering) associated with the tiling $\left\{T_{i}\right\}$.

Throughout this paper, the measure (volume in 3-space or area in the plane) of any set $S$ will be denoted by $|S|$. Also, the length of any segment $s$ will be denoted $|s|$. Each packing (covering) is assigned a nonnegative number, called the density of the packing (covering), 
which, intuitively, corresponds to the ratio of the sum of the measures of the bodies in the packing (covering) to the measure of the region which they pack (cover). For a formal definition of density and an investigation concerning its existence and uniqueness the reader is referred to Section III, Chapter 1 of [2]. The density of a tightest packing of Euclidean space with copies of $K$ is called the packing density of $K$. Likewise, the density of a thinnest covering of Euclidean space with copies of $K$ is called the covering density of $K$. The density of a most efficient packing (covering) with copies of $K$ by a lattice arrangement is the lattice packing (covering) density of $K$. In the case that the packing (covering) $\left\{K_{i}\right\}$ is associated with the tiling $\left\{T_{i}\right\}$, the density of $\left\{K_{i}\right\}$ can be computed as $|K| /|T|$. In general, if $\left\{K_{i}\right\}$ is a packing (covering) and $\left\{T_{i}\right\}$ is a tiling $(i=1,2,3, \ldots)$ and there is a number $r$ such that for every positive integer $i$ the distance between $K_{i}$ and $T_{i}$ is less than $r$, then the density can be computed as $|K| /|T|$. Therefore, if $\left\{K_{i}\right\}$ is a packing (covering) formed by translating copies of $K$ into a lattice arrangement, then the density can be computed as $|K| /|T|$ where $T$ is the lattice parallelepiped.

In this paper the problem of finding a lower bound for the ratio of the packing density to the covering density for an arbitrary convex body is investigated. Such a lower bound can be found for a centrally symmetric $K$ in $E^{d}$ by taking any saturated packing with translates of $K$ and homothetically enlarging each copy about its center by a factor of two. The resulting arrangement must then be a covering, which yields a ratio between packing and covering densities of $2^{-d}$. Here we will prove that for every centrally symmetric convex body $K$ in $E^{3}$, the lattice packing density of $K$ divided by the lattice covering density of $K$ is at least $\frac{1}{4}$. To achieve this, the construction in [5] is used, in which the author shows that every centrally symmetric convex body in $E^{3}$ will pack space with a density of at least $0.46421 \ldots$... An analogous inequality in the plane [4] asserts that the ratio between packing and covering densities for any convex disk is greater than or equal to $\frac{3}{4}$. This result is the best possible, since for a circular disk, the packing density divided by the covering density is equal to $\frac{3}{4}$. In contrast, the result here can probably be improved. For example, the lattice packing density of a sphere in $E^{3}(\pi / \sqrt{18},[3])$ divided by its lattice covering density $(5 \sqrt{5} \pi / 24$, [1] $)$ is $24 / 15 \sqrt{10}=0.50596 \ldots$

\section{The Theorem}

Theorem. If $K$ is a centrally symmetric convex body in $E^{3}$, then its lattice packing density divided by its lattice covering density is greater than or equal to $\frac{1}{4}$.

Proof of the Theorem. We begin by constructing a packing of $E^{3}$ with copies of $K$. For simplicity, we assume that $K$ is strictly convex and has a smooth boundary, since any convex body can be approximated arbitrarily closely by a smooth, strictly convex body. Let $d$ be a chord in $K$ of maximum length. This chord must contain the center of symmetry. Otherwise, there would be two such parallel chords which would imply the existence of a longer chord. Let $L$ and $N$ be planes of support of $K$ such that they each contain an endpoint of $d$. Observe that $d$ is normal to both planes, since otherwise it would not be of maximum length. As a matter of convenience, we say that the orientation of the planes is horizontal and the orientation of $d$ is vertical. Let $o$ be the center of symmetry for $K$ and let $M$ be the plane parallel to $L$ which contains $o$. Let $C$ be the intersection of $M$ with $K$. 


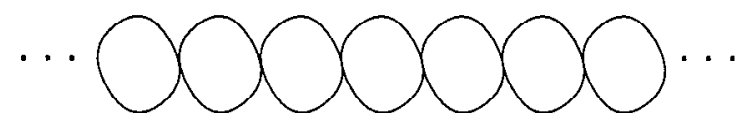

Fig. 1. A row of translates of $C$.

Now we construct a lattice packing of the plane $M$ with copies of $C$ so that each copy touches exactly six other copies. This can be done by choosing a point on the boundary and placing a translate of $C$ so that it touches $C$ only at this point. Also place translates at all integer multiples of the first translational vector to form an infinite row (see Fig. 1). Then take a row of translates identical and parallel to this one and move it so that it lies as closely as possible to the first row while not intersecting its interior. Then $C$ must touch two copies in the other row, since otherwise the row could be shifted by a small amount along its line, allowing the rows to be pushed closer together (see Fig. 2). These two rows define the lattice packing.

Shown in Fig. 3 are $C$ and its translates in the lattice packing of $M$ with their centers at the points of the lattice. We now consider a lattice arrangement of copies of $K$ such that each copy of $C$ is a cross section of a copy of $K$. Assume that $C$ and one of its translates $C^{\prime}$ in this arrangement have a boundary point $p$ in common. $K^{\prime}$ is the translate of $K$ in this arrangement which contains $C^{\prime}$. Because of symmetry, $p$ must be the midpoint of the segment which joins the center of $K$ with the center of $K^{\prime}$. Furthermore, the support plane of $K^{\prime}$ containing $p$ must be parallel to the support plane of $K$ at $p$. Thus the support planes coincide, which implies that the interior of $K$ has no point in common with the interior of $K^{\prime}$. Therefore, this arrangement of translates of $K$ between the planes $L$ and $N$ is a packing.

Our packing of $E^{3}$ will consist of copies of the layer which was just constructed. The layers in the packing will be translates of the first layer. This will be a lattice packing of $E^{3}$, therefore it will only be necessary to look at two adjacent layers in order to understand the entire packing. We denote the original lower layer $\Omega$ and the upper layer $\Omega^{*}$. We arrange the layers so that their supporting planes are parallel and so that $K$ in $\Omega$ touches its counterpart $K^{*}$ in $\Omega^{*}$. Seen in Fig. 4 are dots which represent the projections of segment $d$ and its copies onto the plane $M$. These points correspond to the extreme points or "peaks" in $\Omega$ on either side. Indicated by small circles are the projections of the peaks in $\Omega^{*}$ when they are pushed as far as possible into $\Omega$ without one copy of $K$ penetrating the interior of another. It is easy to observe that $K$ will now be touching at least three copies of itself in $\Omega^{*}$. Otherwise, $\Omega$ could be shifted within its supporting

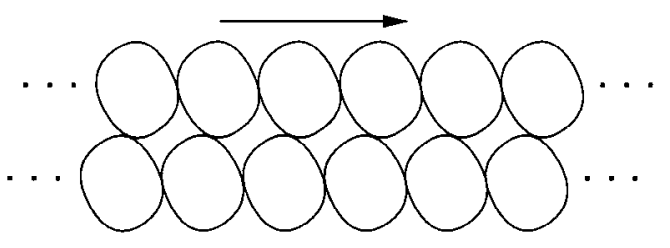

Fig. 2. Two rows which could be packed more closely. 


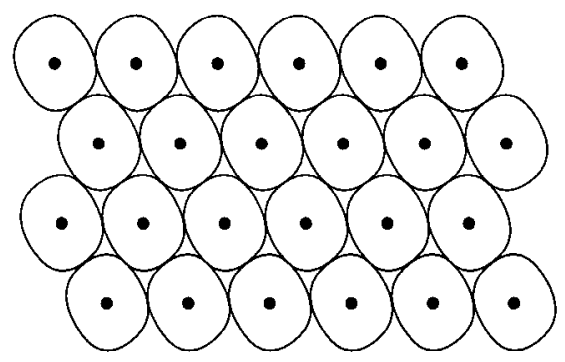

Fig. 3. A packing of $M$ with translates of $C$.

planes in some direction until $K$ does not touch any member of $\Omega^{*}$, allowing the layers to be pushed closer together. Since these are translates of a centrally symmetric convex body, each of these points of contact must be the midpoint of a segment which joins the center $o$ of $K$ with the center of some translate of $K$ in the other layer. Therefore their convex hull must lie in a plane which is parallel to $M$. The triangles formed by the points of contact between each member of $\Omega$ with members of $\Omega^{*}$ are seen in Fig. 5 .

The triangle corresponding to $K$ will be denoted $T_{K}^{1}$. Denote its reflection through the center of symmetry $o$ by $-T_{K}^{1}$. Due to the symmetry of $K$ and of the lattice parallelepiped, $\Omega^{*}$ will be a reflection of $\Omega$ through the midpoint of the segment joining the centers of $K$ and $K^{*}$. Therefore, $K^{*}$ will contain the reflection of triangle $T_{K}^{1}$ through one of its vertices. Figure 6 represents all such triangles in $\Omega^{*}$ (shaded), along with the triangles which were seen in Fig. 5. Notice that the sides of each triangle are parallel to the lattice vectors of a single layer and that each triangle has one-eighth of the area of the lattice parallelogram $P$.

The small circles in Fig. 7 indicate alternate locations for the peaks of $\Omega^{*}$ to be pushed into $\Omega$ so that each copy of $K$ touches three copies of $K$ in $\Omega$ (compare with Fig. 4). These points of contact between layers yield an analogous arrangement of congruent triangles identical to the arrangement in Fig. 4, but rotated $180^{\circ}$ about some point in one of the white triangles and possibly shifted a little (as seen in Fig. 8, with the lighter shading, superimposed upon the first arrangement). We denote the alternate triangle in $K$ by $T_{K}^{2}$. Of these two arrangements of the layers, we choose the one which allows the layers to penetrate each other more deeply, which we have previously assumed

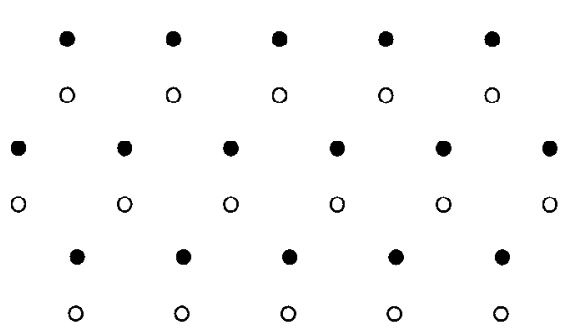

Fig. 4. Projection of the peaks of $\Omega$ and of $\Omega^{*}$. 


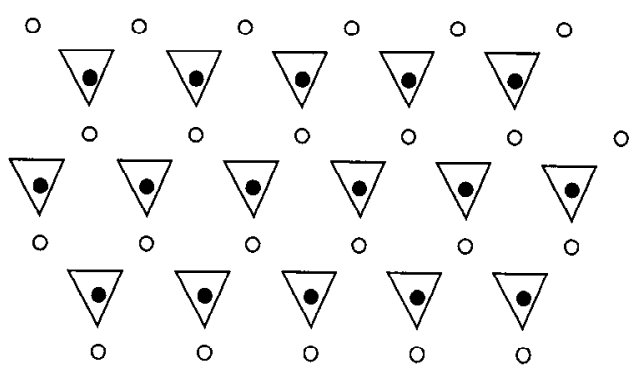

Fig. 5. Convex hulls of contact points for each copy in $\Omega$.

to be the first one. Then the convex hull of $T_{K}^{2}$ with $-T_{K}^{1}$ is a cylinder of triangular base. Its cross section in the plane containing $T_{K}^{1}$ is congruent to $T_{K}^{2}$ and has an identical orientation. The convex hull of this cross section with $T_{K}^{1}$ is a hexagon $H_{K}$, possibly degenerated to a parallelogram. To compute its area, consider the arrangement of the triangles seen in Fig. 9. It is clear that the area of the convex hull is equal to the base of one triangle times its height. If a triangle is translated so that one of its vertices is in the interior of the other triangle, the area of the convex hull will increase. Consequently, the area of the hexagon is at least twice that of one triangle or one-quarter the area of the lattice parallelogram $P$.

Note that $H_{K}$ has its own center of symmetry. Then its reflection $-H_{K}$ through $o$ is identical and in a plane parallel to the plane which contains $H_{K}$. Let $h$ be the vertical distance between $H_{K}$ and $-H_{K}$. Notice that $h$ is also the vertical distance between the centers of the translates in adjacent layers. Therefore, the lattice parallelepiped in this packing has a volume of $h|P|$, implying a density of $|K| / h|P|$.

The convex hull of $H_{K}$ and $-H_{K}$ is a tile of $E^{3}$ and is contained in $K$ having a volume of $h|P| / 4$. Therefore we can cover $E^{3}$ using copies of $K$ with density $|K| /(h|P| / 4)=$ $4|K| / h|P|$. Because of the symmetry properties of the tile, this density can be achieved in a lattice covering. Then the ratio of the lattice packing density of $K$ to the lattice covering density of $K$ is greater than or equal to $(|K| / h|P|) /(4|K| / h|P|)=\frac{1}{4}$.

This completes the proof of the theorem.

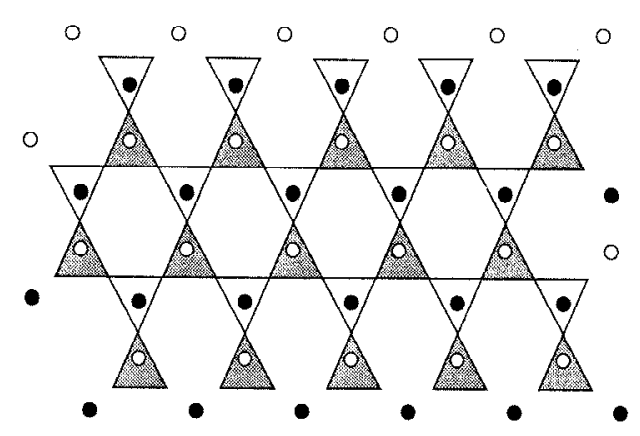

Fig. 6. The triangles of Fig. 5 with each of their reflections in $\Omega^{*}$. 


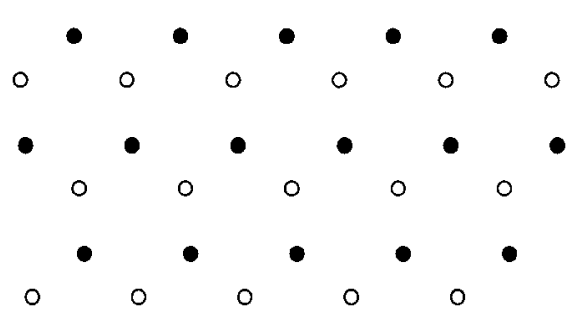

Fig. 7. Alternate locations for the peaks of $\Omega^{*}$.

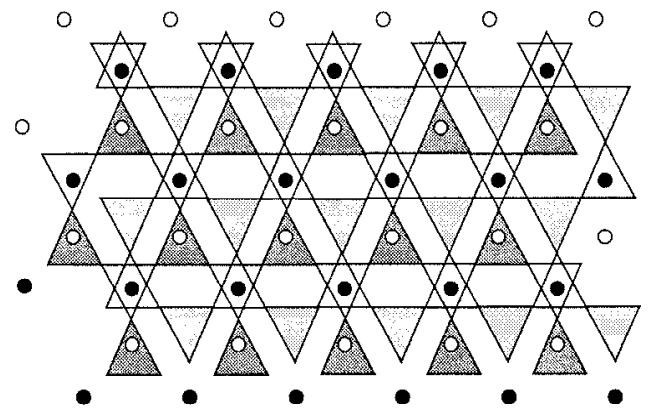

Fig. 8. The triangles for both the original and the alternate arrangements.

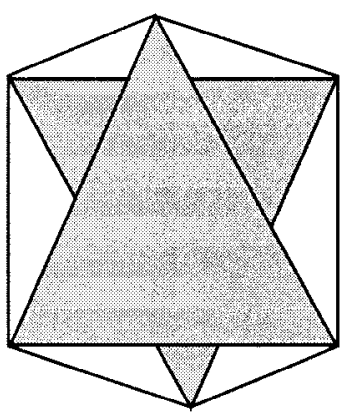

Fig. 9. The area of $H_{K}$ is computed. 


\section{References}

1. R. P. Bambah, On lattice coverings by spheres, Proc. Nat. Inst. Sci. India 20 (1954), 25-52.

2. L. Fejes Toth, Lagerungen in der Ebene, auf der Kugel und im Raum, Springer-Verlag, Berlin, 1972.

3. C. F. Gauss, Untersuchungen uber die Eigenschaften der positiven ternaren quadratischen Formen von Ludwig August Seber, Gottingishe gelehrte Anzeigen, Juli 9 (1831) (J. Reine Angew. Math. 20 (1840), 312-320; Werke, Vol. 2, Konigliche Gesellschaft der Wissenschaften, Gottingen, 1876, pp. 188-196).

4. W. Kuperberg, An inequality linking packing and covering densities of plane convex bodies, Geometriae Dedicata 23 (1987), 59-66.

5. E. H. Smith, A density bound for efficient packings of 3-space with centrally symmetric convex bodies, Mathematika (to appear).

Received October 8, 1998, and in revised form December 30, 1998. 